\title{
Online genetic counseling from the providers' perspective: counselors' evaluations and a time and cost analysis
}

\author{
Ellen Otten $^{\star, 1}$, Erwin Birnie ${ }^{1}$, Adelita V Ranchor ${ }^{2}$ and Irene M van Langen ${ }^{1}$
}

Telemedicine applications are increasingly being introduced in patient care in various disciplines, including clinical genetics, mainly to increase access to care and to reduce time and costs for patients and professionals. Most telegenetics reports describe applications in large geographical areas, showing positive patients' and professionals' satisfaction. One economic analysis published thus far reported lower costs than in-person care. We hypothesized that telegenetics can also be beneficial from the professional's view in relatively small geographical areas. We performed a pilot study in the Northern Netherlands of 51 home-based online counseling sessions for cardiogenetic and oncogenetic cascade screening, and urgent prenatal counseling. Previously, we showed patient satisfaction, anxiety, and perceived control of online counseling to be comparable to in-person counseling. This study focuses on expectations, satisfaction, and practical evaluations of the involved counselors, and the impact in terms of time and costs. Most counselors expected disadvantages of online counseling for themselves and their patients, mainly concerning insufficient non-verbal communication; few expected advantages for themselves. Afterwards, counselors additionally raised the disadvantage of insufficient verbal communication, and reported frequent technical problems. Their overall mean telemedicine satisfaction itemscore was 3.38 before, and 2.95 afterwards, being afterwards slightly below the minimum level we set for a satisfactory result. We estimated reduced time and costs by online counseling with about $8 \%$ and $10-12 \%$, respectively. We showed online genetic counseling to be effective, feasible and cost-efficient, but technical improvements are needed to increase counselors' satisfaction. European Journal of Human Genetics (2016) 24, 1255-1261; doi:10.1038/ejhg.2015.283; published online 20 January 2016

\section{INTRODUCTION}

Telemedicine is increasingly being applied in many medical disciplines, including clinical genetics. Telemedicine applications can be distinguished into remote monitoring (of patient's functions or health), store and forward (transmission of clinical data and electronic images to be analyzed at a later date), and interactive telemedicine (real-time communication between professional and patient). ${ }^{1}$ The reasons for its introduction mainly lie in the potential to improve patient's health, improve access to care - especially for patients in remote areas, and reduction of healthcare costs, for example, by reducing travel time and costs for patients. Moreover, telemedicine applications may increase efficiency for professionals due to spending less time on patient care and/or reduce or even avoid traveling to regional clinics (http://www.americantelemed.org/docs/default-source/ policy/examples-of-research-outcomes-telemedicine's-impact-onhealthcare-cost-and-quality.pdf). ${ }^{1-5}$

Use of telemedicine applications in clinical genetics (commonly called telegenetics) is scarce but may nevertheless be attractive. In clinical genetics, patient numbers rise continuously, due to the introduction and wider availability of new diagnostic techniques, emphasizing the need for increased efficiency and optimization of care within existing personal and financial means. Adaptations to existing care through the introduction of telemedicine may relate to changing the professionals who provide genetic counseling (professional substitution; 'mainstreaming'); changing the content of counseling; or changing the organization or location of counseling (facility or setting substitution). ${ }^{2}$ Depending on the modality chosen, introduction of telemedicine applications may not only have impact on patients and presymptomatic clients, but also on professionals and healthcare costs. Several reports indeed support its favorable cost-effectiveness, (http://www.americantelemed.org/docs/default-source/policy/examplesof-research-outcomes-telemedicine's-impact-on-healthcare-costand-quality.pdf) ${ }^{6,7}$ but also point to technical and privacy issues. ${ }^{1,2}$ Reports on interactive telemedicine application in genetics mainly originate from countries with large traveling distances. They show positive outcomes on (symptomatic and presymptomatic) patients' and counselors' satisfaction with pre-test counseling. ${ }^{8-12}$ The only American publication reporting on the costs of interactive telegenetic pre-test counseling between counselors in central clinics and patients and clients in regional clinics showed comparable satisfaction and less costs compared with in-person counseling. ${ }^{7}$

We performed a pilot study on pre-test online genetic counseling for presymptomatic oncogenetic and cardiogenetic patients and for couples receiving urgent prenatal counseling, being referred to our clinical genetics department in the Northern Netherlands, and compared this with control patients receiving regular pre-test

\footnotetext{
${ }^{1}$ Department of Genetics, University of Groningen, University Medical Center Groningen, Groningen, The Netherlands; ${ }^{2}$ Department of Health Psychology, University of Groningen, University Medical Center Groningen, Groningen, The Netherlands

*Correspondence: Dr E Otten, Department of Genetics, University of Groningen, University Medical Center Groningen, Hanzeplein 1, P.O. Box 30001, Groningen 9700 RB, The Netherlands. Tel: +31 50361 7229; Fax: +31 50361 7231; E-mail: e.otten@umcg.nl

Received 17 July 2015; revised 29 October 2015; accepted 8 December 2015; published online 20 January 2016
} 
in-person presymptomatic and prenatal genetic counseling at the outpatient department (OPD). ${ }^{13}$ Our telegenetics application was an example of interactive telemedicine, substituting the setting of care by introducing online genetic counseling at patients' homes instead of counseling at the OPD of our university center, with the same genetic professionals and content of care as in conventional genetic counseling, as current professional guidelines and the professionals' view recommend. ${ }^{14,15}$ The results showed that our application was feasible that patients were satisfied with the online counseling, with its efficiency, and with the specific online application we used. Levels of anxiety and personal perceived control of online patients and controls were comparable, thereby demonstrating equivalence of online counseling and regular OPD counseling from the patients' perspective.

In the current study, we report the impact of our online counseling application on counselor's satisfaction with telemedicine, their evaluation of practical aspects of online counseling, and the impact on time and costs of counseling compared with conventional OPD counseling. Knowing whether counselors are willing to accept this new modality, and identifying any barriers, could support successful implementation. We hypothesized that telegenetics could offer benefits for professionals regarding increased efficiency and flexibility, and lower costs, even in our small country.

\section{METHODS}

\section{Study setting, design, and participants}

In The Netherlands, clinical genetic care is concentrated in eight university medical hospitals, spread throughout the country, and is covered by health insurances. Our genetics department of the UMC Groningen is the only one in the Northern Netherlands (area about $8300 \mathrm{~km}^{2}$ and 1.7 million inhabitants), and holds regular outpatient clinics in five regional hospitals (maximal travel distance of $125 \mathrm{~km}$ ).

We performed a cohort study with measurements before, during, and after the study for 10 participating counselors between November 2011 and June 2012. They represented an average of the total counselors group of our department with regard to age, sex, profession, and attitude toward online counseling. These counselors performed the pre-test online sessions instead of in-person sessions for oncogenetic or cardiogenetic cascade screening for a known familial mutation, and urgent prenatal counseling with an appointment planned at least 2 days after referral. Online sessions were performed at the department while patients were at their homes. Patients were eligible if they had access to a computer with internet and a webcam, and gave consent. No more than two participants at the patient's side could participate per online session. If not interested, not suitable, or not equipped for online counseling, patients were scheduled for regular in-person counseling. Counselors were asked to do at least one session from their homes, to test the intended flexibility of online counseling. We recorded counselors' expectations, their satisfaction with telemedicine, and evaluation of practical issues at baseline, after each counseling session, and at the end of the pilot period, and we estimated the costs of providing this type of care. The institutional medical ethics committee declared that the study protocol was exempted from formal review (number M11.108133)

\section{Online counseling application}

Counseling sessions were performed through the online platform 'myCoachconnect' (www.mycoachconnect.com), which was adapted for use in our clinical genetics practice and met the UMC's required safety protocols. Privacy protocols were composed in cooperation with the hospital's ICT and personal privacy protection departments. In addition to videoconferencing, the platform offered several functions intended to support the information exchange during counseling, and to allow for future access to this information for the patients: this included online file notes for counselors and a notebook for patients, the transfer and viewing of disease brochures, visiting of websites by counselor and patient together during counseling, and secured email (Supplementary Figure 1a-d). Before the study onset, all 10 counselors were trained in a workshop and could limitless practice individually in order to become familiar with the online application. Support was provided by a web coordinator, researcher (EO), and case managers. An ICT technician and the myCoachconnect helpdesk were available for supportive services and immediate assistance to the professionals whenever needed.

\section{Online counseling procedures}

Consenting patients were evenly allocated to the counselors in order of referral, taking into account counselors' availability and subspecialism. New patients were allocated to the counselor who had previously seen their family members, whenever possible.

Genetics professionals had to prepare as follows before an online counseling session could take place: (1) indicating the counselors' preferred date and time for the session, (2) checking if any additional medical information had to be gathered, (3) registering patients' personal and appointment information on the administrator-side of the application (Supplementary Figure 1e). This automatically generated an email to the patient, including their appointment information, account/login instructions, and a link to an instruction movie. Patients had to complete and return a digital form with family information after creating an account. (4) Processing the received family information forms to update/expand existing family pedigrees. (5) Contacting patients to test the connection a few days before their session. (6) Preparing and sending test kits/ forms to patients for them to submit saliva or blood samples for DNA testing after counseling. Overall, the preparations for online counseling were about the same as for OPD counseling, but contained more digital than paper process steps and consequently slightly less administrative time. The researcher and case managers monitored the whole process and contacted patients when necessary. After each online session, counselors made their usual report in the patient's regular paper medical record.

\section{Determinants and outcomes}

We used online questionnaires to assess counselor specific determinants at the start of the study. Outcomes were assessed immediately after each online session and at the end of the pilot study. ID codes were used to identify each counselor. The determinants and outcome measures we assessed were:

Counselor and counseling characteristics. Date, time, and type of counseling (oncogenetic, cardiogenetic, prenatal), location of counseling (at the department, at home, elsewhere), and the sequence number of the online session performed by the counselor.

Experience with computers, internet, and online communication. Counselors were asked to indicate their time spent using internet for private purposes, and their experiences and frequency of use of different online platforms both at the start and end of the pilot project. Also, on both time points, their experience with computer use and online communication were recorded, by rating four statements on a five-point Likert scale ('totally disagree' to 'totally agree'): (1) 'always looking for new possibilities on my computer'; (2) 'expecting to increase working via webcam communication'; (3) 'annoying to use a computer when counseling patients' and (4) 'I imagine patient feels annoyed when being counseled via a computer'.

Expectations and evaluations of online counseling. Counselors were asked to describe their expected and observed advantages and/or disadvantages of online counseling over in-person counseling at the OPD, both for themselves and their patients. They were also asked to indicate if and how online counseling had to be improved to make it equivalent to OPD counseling.

Telemedicine Satisfaction Questionnaire. The Telemedicine Satisfaction Questionnaire (TSQ) aims to measure patient satisfaction with telemedicine regarding quality of care and comparison of online care to in-person care. ${ }^{16} \mathrm{We}$ translated the validated 14-item English version into Dutch and adapted it for use amongst counselors (Supplementary Figure 2) to measure both their expected satisfaction with telemedicine before the pilot project, and their observed satisfaction after each counseling session, and at the end of the pilot. We removed item seven ('I think the healthcare provided via telemedicine is consistent') of the original questionnaire because this was inapplicable in our 
setting. ${ }^{16}$ Response mode was a $1-5$ Likert scale, higher scores indicate higher satisfaction. Internal consistency (Cronbach's alpha) was 0.88 and 0.72 for the TSQ before and TSQ after counseling, respectively. We considered the counselor's evaluation successful when the satisfaction with telemedicine was at least 'neutral' (ie, score $\geq 3$ ), and the application functioned sufficiently in technical respect.

Technical problems. The occurrence and type of technical problems during online counseling were recorded.

Patient responsibilities. Counselors were asked to indicate the degree they accepted any responsibility for patients in the online counseling process on a 1-5 Likert scale ('not acceptable at all' to 'very acceptable') regarding: (1) planning an appointment, (2) doing preparations before counseling, (3) proper functioning of the application during counseling, and (4) self-collection of saliva.

\section{Patient evaluations; analysis of time and cost}

In our previous report on this pilot project, we demonstrated that the psychological outcomes (anxiety and perceived personal control, measured with the STAI and PPC questionnaires) of patients who received online counseling and control patients were comparable. ${ }^{13}$

As a result, we adopted a cost-minimization analysis as our main analytical framework. This implied that we compared the total time and costs spent on the process of online counseling to the in-person counseling process. First, for each stage in the process of online counseling (Figure 1), we estimated the total time spent by counselors, administrative staff, and the pilot study team. The time spent by the pilot team members only included care-related time; the time and costs of research-related activities were excluded. For each professional, the average time spent on conventional OPD counseling per stage was obtained from a detailed workflow and process time sheet. The total time spent on the process of online counseling was extrapolated from the same sheet and added with specific time registrations/measurements and interviews. Second, time of professionals was valued at their gross hourly wage (excluding departmental and hospital overheads).

\section{Analysis}

We used the mean, SD and range as descriptive statistics for quantitative variables and $n(\%)$ for nominal and ordinal variables. Mean TSQ item scores within counselors before versus after the pilot study were quantified as the effect size, defined as the difference in mean TSQ score before and after counseling, divided by the pooled SD of these mean TSQ scores. An effect size of 0.50 is considered a relevant difference. ${ }^{17}$ There were no missing TSQ items. The profiles of counselor's mean TSQ item scores over the successive online sessions during the pilot study were compared with repeated measurements analysis (mixed linear modeling). The dependent variable was the profile of mean TSQ item scores; the repeated factor was the counseling session (covariance structure: unstructured); and the covariables were the mean TSQ itemscore at baseline (before the pilot), counselor-specific characteristics (age, gender, profession, years of experience) and counseling-specific characteristics (whether the online counseling was held in the office or at home, whether the counseling took place during/outside office hours, and if a technical problem occurred during the session). Pre-TSQ values and the counseling number were entered in the analysis; the other determinants were analyzed in a backward stepwise procedure. Analyses were performed with SPSS statistics v22, IBM Corporation, NY, USA.

\section{RESULTS}

\section{Counselors and patient's characteristics}

Ten counselors performed a total of 51 counseling sessions with 57 patients, including 16 sessions conducted from counselors' homes. The average number of sessions per counselor was 5 (range 2-7): one prenatal counselor did not perform all five predetermined sessions. Three pairs of patients joined one session for oncogenetic counseling; three couples (six patients) participated in prenatal counseling (Supplementary Table 1 for detailed counselors' and patients' characteristics).

\section{Counselors' experiences with computers, internet, and online communication}

At the start of the pilot, counselors spent from less then $30 \mathrm{~min}$ to $2 \mathrm{~h}$ per day on the internet for private purposes, and seven counselors had experience with online communication in a manner other than by sending email (eg, skype, social media). After the pilot, the time spent on the internet overall had increased slightly, and eight instead of seven counselors by then had experience with online communication other than email.

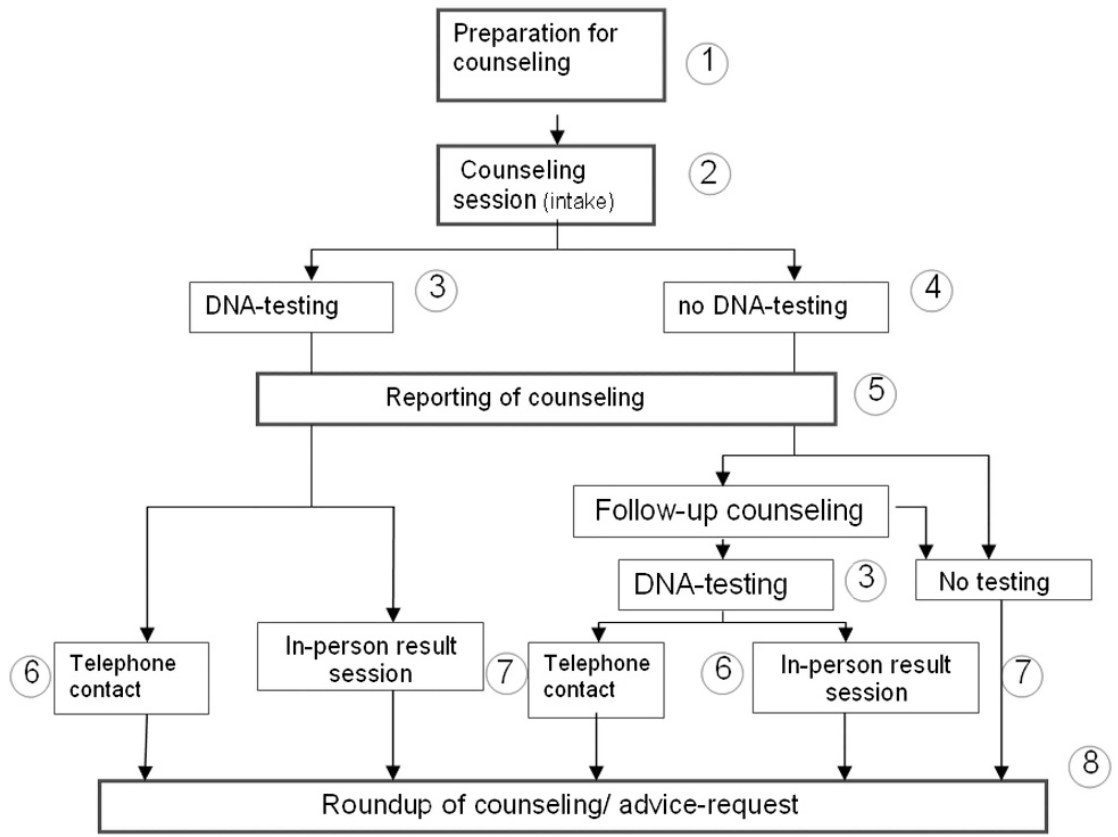

Figure 1 Overview of the general process of online counseling and testing. A full color version of this figure is available at the European Journal of Human Genetics journal online. 
The counselors group overall scored the four multiple choice questions regarding computer use all neutrally, both before and after the pilot (mean overall score before 3.1; after 3.4; range 1-5). Interestingly, the individual changes reflected a considerable disparity among the counselors, and 5 of 10 counselors actually experienced less annoyance themselves, and imagined less annoyance on their patients' behalf, compared with the level of annoyance they had expected beforehand on using a computer for performing genetic counseling.

\section{Expectations and evaluation of online counseling aspects}

Table 1 shows that before the start of the pilot, few counselors saw any advantages to online counseling above in-person counseling for themselves (three counselors; four advantages), whereas most of them saw any disadvantages for themselves (7 counselors; 12 disadvantages). Moreover, for their patients, most counselors saw some advantages (9 counselors; 17 advantages), but also disadvantages (8 counselors; 12 disadvantages) of online counseling. The counselors saw the time and/ or cost savings by not having to travel as the main advantage of online counseling both for their patients and for themselves. The expected disadvantages for their patients and themselves mainly concerned the reduced quality of non-verbal communication in online sessions.

After the pilot period, counselors reported a larger number of advantages (8 counselors; 9 advantages) and disadvantages (10 counselors; 20 disadvantages) for themselves than before the pilot. Qualitatively insufficient verbal communication was being raised as an additional disadvantage that was not reported beforehand. The number of advantages and disadvantages and the balance between these, as seen by the counselors for their patients, remained about the same (Table 1). The improvements to the online counseling system proposed by nine of the 10 counselors - to make it more equal to in-person counseling - all concerned technical aspects of the application: for example, solving the sound delays and echoes during counseling, providing a larger and clearer webcam image on screen.
Two counselors stated that online counseling could never become equal to in-person counseling.

\section{Telemedicine satisfaction questionnaire}

Figure $2 \mathrm{a}$ and $\mathrm{b}$ show the mean TSQ item scores for each individual counselor before, during (after each session) and after the pilot. The mean TSQ item score afterwards for the average counselor was somewhat lower than before the pilot, and slightly below the minimum level we set beforehand for a successful outcome (mean (SD) score before: 3.38 (0.68) vs after: 2.95 (0.96); 1-5 scale; Figure 2a). The effect size was 0.52 , indicating a relevant change. There were individual differences among counselors, since seven counselors had higher mean TSQ item scores afterwards compared with before, while three had lower scores afterwards.

Figure $2 \mathrm{~b}$ shows the variability in the profiles of mean TSQ item scores among the counselors and over their successive counseling sessions. For 17 of $51(33.3 \%)$ counseling sessions, the mean TSQ itemscore was $<3$. The impact on the profile of mean TSQ item scores was as follows: counselor's mean TSQ item score before the pilot onset: beta $=0.67$ (95\% CI: $0.30-1.04)$; presence of a technical problem: beta $=0.04$ (95\% CI: -0.16 to 0.23$)$; and number of counseling session: beta $=0.09$ per extra counseling, 95\% CI: $0.03-0.15)$, implying that the mean TSQ itemscore increased on average when more online sessions were performed. These impacts remained about the same when other characteristics of the counselor and counseling session were added; especially location of counseling at department instead of own home (beta $=0.21,95 \% \mathrm{CI}$ : -0.34 to 0.77 ), female gender (beta $=0.34,95 \%$ CI: -0.28 to 0.97 ) and counseling during instead of outside office hours (beta $=-0.66$, 95\% CI: -1.60 to 0.28$)$. The impact of counselor's age and professional experience was small.

Looking at the individual items, the worst-scoring after the pilot were items 1 and 4 'I could easily talk to my patient' (mean score 2.4) and 'I could see my patient as if we met in person' (mean score 1.8).

Table 1 Advantages and disadvantages of online counseling mentioned by counselors before and after the online counseling period

\begin{tabular}{|c|c|c|c|c|}
\hline \multirow[b]{2}{*}{ Advantages } & \multicolumn{2}{|c|}{ Before } & \multicolumn{2}{|c|}{ After } \\
\hline & $\begin{array}{l}\text { For counselor } \\
\qquad(n=3)^{a}\end{array}$ & $\begin{array}{l}\text { For patient } \\
\qquad(\mathrm{n}=9)^{a}\end{array}$ & $\begin{array}{l}\text { For counselor } \\
\qquad(\mathrm{n}=8)^{\mathrm{a}}\end{array}$ & $\begin{array}{l}\text { For patient } \\
\qquad(\mathrm{n}=10)^{\mathrm{a}}\end{array}$ \\
\hline Time/cost saving; avoid traveling & 2 & 9 & & 10 \\
\hline Increased flexibility & 2 & 3 & 4 & 2 \\
\hline Less stressful in case of illness or disability & & 2 & & \\
\hline Better grip on family/multiple family members counseled by same counselor & & 1 & 1 & 1 \\
\hline Counseling in familiar environment & & 1 & & 2 \\
\hline Possibility of watching counseling session again & & 1 & & \\
\hline Disadvantages & $\begin{array}{l}\text { For counselor } \\
\qquad(\mathrm{n}=7)^{a}\end{array}$ & $\begin{array}{l}\text { For patient } \\
\qquad(\mathrm{n}=8)^{\mathrm{a}}\end{array}$ & $\begin{array}{l}\text { For counselor } \\
\qquad(\mathrm{n}=10)^{\mathrm{a}}\end{array}$ & $\begin{array}{c}\text { For patient } \\
(\mathrm{n}=10)^{\mathrm{a}}\end{array}$ \\
\hline Less non-verbal communication & 6 & 5 & 7 & 3 \\
\hline Less extensive information/verbal communication & & & & 4 \\
\hline Greater role for/attention to technique & 3 & 4 & 1 & 2 \\
\hline Less notion of interaction at patients'side & 1 & & & \\
\hline Less personal contact & 1 & 3 & 2 & 1 \\
\hline More working from behind PC & 1 & & & \\
\hline Insufficient verbal communication & & & 7 & \\
\hline Less easy discussing psychological aspects & & & 3 & 1 \\
\hline
\end{tabular}

Numbers in the table reflect the number of times the particular advantages and disadvantages are being mentioned by counselors.

a (within brackets) the number of counselors included. 
a mean TSQ itemscore per counselor

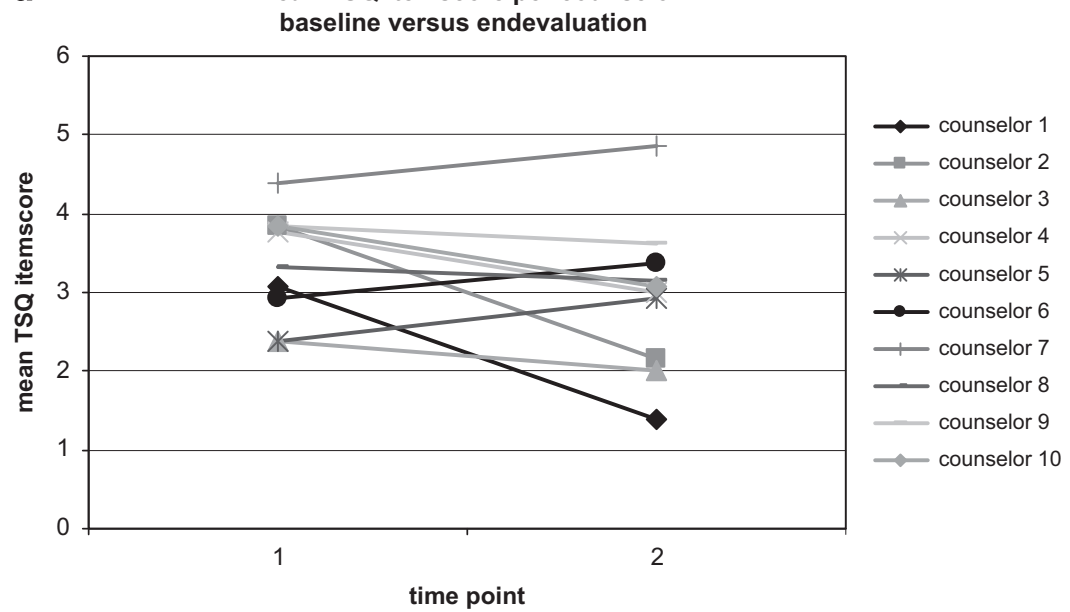

b course of mean TSQ itemscore per counselor per successive session

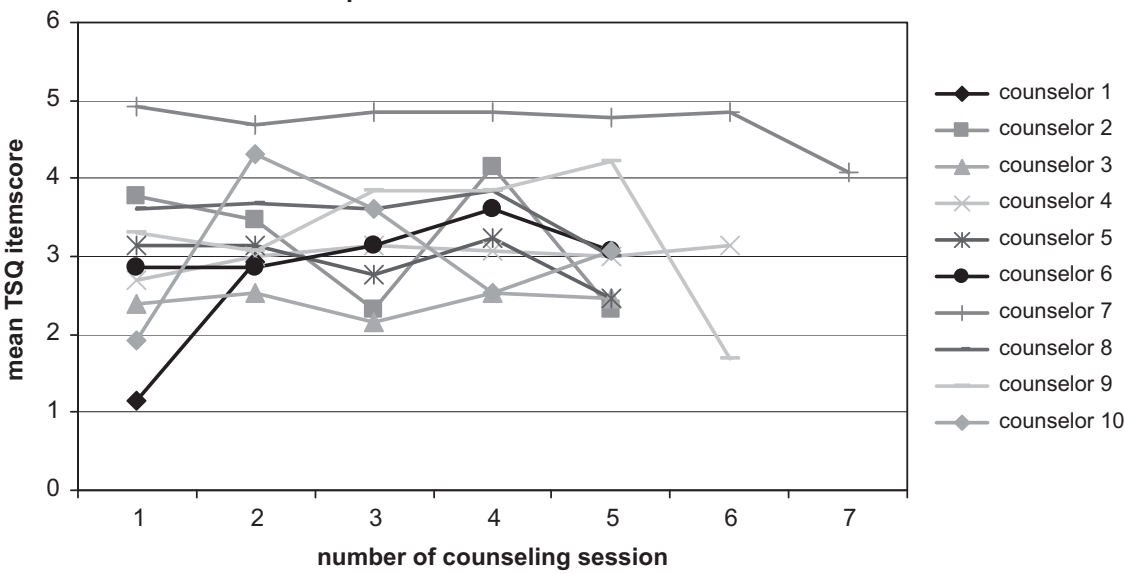

Figure 2 Mean TSQ itemscores per counselor, baseline versus endscores (a) and for their successive counseling sessions (b). A full color version of this figure is available at the European Journal of Human Genetics journal online.

Table 2 Time investments (average per patient) and costs (average per patient, in $€$ ) for each process step by counseling type (in-person, online) and indication (cardiogenetic/oncogenetic, prenatal)

\begin{tabular}{|c|c|c|c|c|}
\hline Process step & In person/OPD counseling & Online counseling & In person/OPD counseling & Online counseling \\
\hline 1. Preparation & 159.5 & 155.0 & 130.5 & 133.0 \\
\hline \multicolumn{5}{|l|}{ 2. Counseling } \\
\hline Traveling & 20.0 & None & 20.0 & None \\
\hline 5. Telephone contact & 4.0 & 4.0 & 4.0 & 4.0 \\
\hline $7+8$. In-person/online result session, round up ${ }^{a}$ & 30.0 & 34.0 & 30.0 & 30.0 \\
\hline Total time (min) & 401.0 & 370.5 & 430.5 & 392.5 \\
\hline Total costs $(€)$ & $€ 361.22$ & $€ 324.26$ & $€ 379.33$ & $€ 332.38$ \\
\hline
\end{tabular}

Abbreviation: OPD, outpatient department.

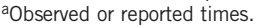

The best-scoring items were items 3 and 7: 'I was able to understand the healthcare condition of my patient' (mean score 4.3) and 'I think I rendered my patient a service by offering telemedicine' (mean scores 3.7; Supplementary Figure $3 \mathrm{a}$ and $\mathrm{b}$ ).
Technical problems

In total, counselors reported technical problems in 26 of 51 (51\%) sessions, of which 9 were performed from their homes. Problems were related to sound (no sound/too soft sound, echo, delayed sound; 19 
sessions (37\%)), internet connection (no or broken connection between counselor and patient; 5 sessions (10\%)); and webcam image (no image/frozen image; 7 sessions (14\%)). Additional remarks referred to the technical imperfections of the online system, as well as its instability, that is, the sound delay was sometimes reported to be as high as several seconds, greatly hampering the conversation, while at other times there was hardly any delay or echo.

\section{Patients' responsibilities}

The assignment of shared responsibilities to patients in the online counseling process was found overall acceptable by the counselors for all the given aspects: establishing an appointment by email (mean score 4.8); performing preparatory actions (mean score 3.9); well-functioning system during the session (mean score 4.3); selfcollection of saliva for DNA testing (mean score 4.4; score range 1: highly unacceptable to 5: highly acceptable).

\section{Time and cost analysis}

Table 2 compares the costs and time per patient spent for online counseling and in-person counseling. The estimated time savings from online counseling for the professionals were $7.6 \%$ for cardiogenetic/ oncogenetic indications and $8.8 \%$ for prenatal indications. The cost savings from online counseling for the professionals were $10.2 \%$ for cardiogenetic/oncogenetic indications and $12.4 \%$ for the prenatal group. The type of counseling did not significantly affect the uptake rate of DNA testing (online group: 54/57 (95\%) vs in-person group: $66 / 71$ (93\%); $P=0.73)$. Follow-up showed that healthcare use after rounding of the counseling was rare in both groups (online group $1 / 57(1.8 \%)$ vs in-person group $2 / 71 \quad(2.8 \%) ; P=0.99)$. Potential cost differences arising by these two factors hardly affect the savings of time and costs in Table 2.

\section{DISCUSSION}

Our results show that, based on the effect size of the change, counselor satisfaction with telemedicine after the pilot was decreased compared with their baseline expectations, being slightly below the minimum acceptable level we had set prior to the study. We also found a large variation in TSQ scores among and within counselors for their start and end scores, and for their scores after each session. Counselors' overall attitudes towards digital communication remained about the same over the two measurements. After the pilot, the number of advantages and disadvantages of online counseling seen by counselors was more balanced than beforehand. They reported flexibility and cost and time savings as the main advantages, and insufficient verbal and non-verbal communication as the main disadvantages. Substantial improvements on the technical side of the application were considered a prerequisite by counselors for implementing online counseling in regular patient care. This was supported by their reports of technical problems in half of the counseling sessions. Counselors found the level of patient responsibilities for online counseling acceptable. Finally, our time and cost analysis showed that online counseling involved less time and lower costs for professionals than in-person counseling.

Our study was a cohort study on online pre-test genetic counseling for presymptomatic clients, in which we only changed the setting of care and maintained the involved professionals and the content of care. By doing so, we could validly compare if the change of setting in online counseling would deliver comparable outcomes with our regular care provision, excluding any possible effect from change of professionals or content of care. The characteristics of our online application made it remote but similar to face-to-face. Nevertheless, several limitations could have influenced our results: (1) counseling sessions were performed for a relatively favorable patient group, who consented to online counseling, and thus might have been more open and cooperative than average. This selection allowed for a good evaluation of counselor's experiences, but their evaluations might be less favorable if they had to counsel less well-motivated patients. (2) We specifically and deliberately included presymptomatic patients and only few but prevalent types of indications. Offering online counseling for symptomatic patients and other indications could also influence the counselors' evaluations. (3) The counselors' questionnaires included mainly non-validated measures, which could have led to biased results. However, the results of the various non-validated measures were largely in agreement with each other. Specifically, we used an adapted TSQ for measuring counselors' satisfaction. Its use for counselors, and the resulting outcomes may be invalid.

We recognize that the number of counselors and sessions was relatively small. It is unlikely that a larger group of counselors could influence the results, since they already represented a mix of gender, age, and experience with computers and counseling, and their attitude beforehand was average. Only one counselor left the pilot study prematurely because of negative experiences. It seems equally unlikely that the number of sessions played a role: in view of the dominant role of technical problems, more sessions would probably not have led to different insights. In the future, it may be worthwhile to study counselor's evaluations again when the application is adapted to the problems reported.

The limitations in personal contact and non-verbal communication as reported by our counselors' are in agreement with previous reports. ${ }^{10,12,18}$ However, in the previous reports counselors were positive overall about online counseling despite the limitations, whereas our counselors were less satisfied with online counseling. ${ }^{10,12,18}$ Our counselors reported many technical problems, which was reflected in their TSQ scores. Improvements and adjustments to the quality and design of the online system are needed, and may contribute to improved counselors' satisfaction in the future. Recently, we piloted an alternative platform to be used in regular patient care, and the first experiences are promising.

Various explanations might underlie the differences between our counselors and the counselors in previous reports. First, our online approach and system differed from previous reports: (1) there was no additional counselor on site with the patient supporting the counseling process, as in previous studies; $8,11,18,19$ (2) counselors used laptops with built-in webcams, and patients used their own equipment at home to run the online application instead of using videoconferencing equipment and television screens; $;^{8,10,18}$ and (3) supportive tools were shown on-screen together with the webcam image, which was an advantage of our system, but the patient's image was small and not 'whole-screen' visible to the counselor.

Second, in previous reports, online counseling mainly enabled counselors to avoid traveling large distances, thereby saving them a significant amount of time. ${ }^{7,9,10}$ This advantage might imply that they were more easily satisfied with online counseling than the counselors in our study in a non-remote area, having a less substantial benefit from avoiding traveling and less time saved. In contrast to this, several of our counselors reported that online counseling allowed for increased flexibility, by using counselors' and patients' laptops/PCs rather than the relatively static videoconferencing systems. Also, our online counseling extends the number of counselor's tasks that could be done from their homes. Our all-in-one online system has not been reported before, and only one report thus far stated that patients' own home equipment was used. Regrettably counselor's experiences with that system were not reported. ${ }^{9}$ 
Finally, we saw a discrepancy between our counselors' satisfaction and our previously reported patients' satisfaction levels, ${ }^{13}$ with the counselors being less satisfied. This is likely due to differences in expectations and requirements by the two groups: while patients are likely to see online counseling as an advantage beforehand (no need to travel, being in own environment), counselors are used to conventional counseling at the OPD, and to the generally accepted, and their own standards for and experiences with good quality of counseling. ${ }^{20-22}$ The counselors also did multiple online sessions and were thus more vulnerable to repeated technical problems, whereas patients only had one session.

This contrast between our counselors and patients also points to the issue whose opinion is most important in evaluating the value of this new counseling option in regular care, and whether the patients' positive evaluations justify that professionals' reported drawbacks should be neglected. It also raises the question of 'what is good care?' Our counselors' moderate satisfaction with online counseling does not necessarily imply that the provision of counseling was suboptimal: most of the identified key aspects of genetic counseling reported by Rantanen et al. were met, ${ }^{23}$ and thus could objectively be regarded as 'good care'. However, we acknowledge that greater counselor satisfaction, for example, by technical improvement of the system, by creating increased awareness of the potential advantages, and perhaps by increasing their experience or familiarity with it, would further increase the acceptance of this new option, as several applied theories suggest. ${ }^{24}$ This would in turn contribute to its implementation in regular patient care.

Online counseling results in time and cost savings for professionals, in addition to the acceptable patient outcomes as reported previously. ${ }^{13}$ Therefore, online counseling can be regarded an adequate additional way of counseling in regular clinical genetic care, although one should be cautious about the saving in time and costs that can be realized in practice. In view of the increasing patient numbers it is likely that any time and costs saved will be devoted to new patients. Additional time saving could be obtained by avoidance of traveling to the hospital each working day when online counseling is performed from the counselor's own home. By offering online counseling as a care modality alongside regular OPD counseling and not as a complete substitute, the eventual efficiency gain will be less evident. On balance, introducing online counseling could still be beneficial, since more patients can be counseled within the existing staff capacity. Finally, when recontacting former patients becomes part of regular patient care, as we expect it will in the near future, the use of online systems can support this process, with patients being given the new information in an online session..$^{25,26}$

To conclude, the implementation of online counseling in regular care potentially can be attractive for patients as well as for counselors, provided that the online application is technically improved for use in regular care. Our way of online counseling leads to more flexibility for counselors, to lower costs and less time investment compared with conventional counseling. It can complement existing genetic counseling services and be offered to part of our patients as an additional service, even in relatively small geographical areas.

\section{CONFLICT OF INTEREST}

The authors declare no conflict of interest.

\section{ACKNOWLEDGEMENTS}

This pilot project was facilitated by grants from the Dutch 'Innovatiefonds zorgverzekeraars' and from the European Social Fund (Grant number
2010ESFN630). We thank the counselors, case managers, web coordinator, Theo van de Meer (De Praktijk), and Jack van Gils and the other contributors from his company myCoachconnect for their participation and support. We thank Jackie Senior for editing our manuscript.

1 Flodgren G, Rachas A, Farmer AJ, Inzitari M, Shepperd S: Interactive telemedicine: effects on professional practice and health care outcomes. Cochrane database Syst Review 2015; 9: CD002098.

2 Silva BMC, Rodrigues JJPC, De la Torre Díez I, López-Coronado M, Saleem K: Mobilehealth: a review of current state in 2015. J Biomed Inform 2015; 56: 265-272.

3 Mehrota A, Paone S, Martich GD, Albert SM, Shevchik GJ: Characteristics of patients who seek care via evisits instead of office visits. Telemed J E Health 2013; 19: 1-5.

4 Merell RC: Editorial: Telemedicine for vulnerable populations. Telemed J E Health 2014; 20: 885-886.

5 Stalker HJ, Wilson R, McCune H, Gonzalez J, Moffett M, Zori RT: Telegenetic medicine: improved access to services in an underserved area. J Telemed Telecare 2006; 12: $182-185$.

6 Wade VA, Karnon J, Elshaug AG, Hiller JE: A systematic review of economic analyses of telehealth services using real time video communication. BMC Health Serv Res 2010; 10: 233.

7 Buchanan AH, Datta SK, Skinner CS et al: Randomized trial of telegenetics vs. in-person cancer genetic counseling: cost, patient satisfaction and attendance. J Genet Counsel 2015; 24: 961-970.

8 Zilliacus E, Meiser B, Lobb AE et al: Are videoconferenced consultations as effective as face-to-face consultations for hereditary breast and ovarian cancer genetic counseling? Genet Med 2011; 13: 933-941.

9 Meropol NJ, Daly MB, Vig HS et al: Delivery of internet-based cancer genetic counseling services to patients' homes: a feasibility study. J Telemed Telecare 2011; 17: $36-40$.

10 d'Agincourt-Canning L, McGillivray, Panabaker K et al: Evaluation of genetic counseling for hereditary cancer by videoconference in British Columbia. BC Med J 2008; 50: 554-559.

11 Elliot AM, Mhanni AA, Marles SL et al: Trends in telehealth versus on-site clinical genetics appointments in Manitoba: a comparative study. J Genet Couns 2012; 21: 337-344

12 Zilliacus E, Meiser B, Lobb E, Dudding TE, Barlow-Stewart K, Tucker K: The virtual consultation: practitioners' experiences of genetic counseling by videoconferencing in Australia. Telemed J E Health 2010; 16: 350-357.

13 Otten E, Birnie E, Ranchor AV, Van Langen IM: Telegenetics use in presymptomatic genetic counseling: patient evaluations on satisfaction and quality of care. Eur J Hum Genet 2016; 24: 513-520.

14 Skirton H, Goldsmith L, Jackson L, Tibben A: Quality in genetic counseling for presymptomatic testing - clinical guidelines for practice across the range of genetic conditions. Eur J Hum Genet 2013; 21: 256-260.

15 Bensend TA, McCarthy Veach P, Niendorf KB: What's the harm? Genetic counselor perceptions of adverse effects of genetic service provision by non-genetics professionals. J Genet Counsel 2014; 23: 48-63.

16 Yip MP, Chang AM, Chan J, Mackenzie AE: Development of the Telemedicine Satisfaction Questionnaire to evaluate patient satisfaction with telemedicine: a preliminary study. J Telemed Telecare 2003; 9: 46-50.

17 Hojat M, Xu G: A visitor's guide to effect sizes: statistical significance versus practical (clinical) importance of research findings. Adv Health Sci Educ Theory Pract 2004; 9 : 241-249.

18 Iredale R, Gray J, Murtagh G: Telegenetics: a pilot study of video-mediated genetic consultations in Wales. Int J Med Market 2002; 2: 130-135.

19 Zilliacus E, Meisser B, Lobb E, Barlow-Stewart K, Tucker K: A balancing acttelehealth cancer genetics and practitioners' experiences of a triadic consultation. J Genet Couns 2009; 18: 598-605.

20 Whitten P, Love B: Patient and provider satisfaction with the use of telemedicine: overview and rationale for cautious enthusiasm. J Postgrad Med 2005; 51: 294-300.

21 Grigsby B, Brega AG, Bennett RE et al: The slow pace of interactive video telemedicine adoption: the perspective of telemedicine program administrators on physician participation. Telemed J E Health 2007; 13: 487-499.

22 Barton PL, Brega AG, Devore PA et al: Specialist physicians' knowledge and beliefs about Telemedicine: a comparison between users and nonusers of the technology. Telemed J E Health 2007; 13: 487-499.

23 Rantanen E, Hietala M, Kristoffersson $\mathrm{U}$ et al: What is ideal genetic counseling? A survey of current international guidelines. Eur J Hum Genet 2008; 16: 445-452.

24 Yi MY, Jackson JD, Park JS, Probst JC: Understanding information technology acceptance by individual professionals: toward an integrative view. Inform Manage 2006; 43: 350-363.

25 Aronson SJ, Clark EH, Varugheese M, Baxter S, Babb LJ, Rehm HL: Communicating new knowledge on previously reported genetic variants. Genet Med 2012; 14 713-719.

26 Hunt $\mathrm{K}$ : Individualizing the informed consent process for whole genome sequencing A patient directed approach [PhD thesis]. AZ, USA: Arizona State University, 2013.

Supplementary Information accompanies this paper on European Journal of Human Genetics website (http://www.nature.com/ejhg) 